\title{
Exploring a Bilingual Next Word Predictor for a Federated Learning Mobile Application
}

This paper was downloaded from TechRxiv (https://www.techrxiv.org).

\section{LICENSE}

CC BY 4.0

SUBMISSION DATE / POSTED DATE

08-01-2022 / 14-01-2022

\section{CITATION}

Burgos, Natali Alfonso; Kiš, Karol; Bakarac, Peter; Kvasnica, Michal; Licitra, Giovanni (2022): Exploring a Bilingual Next Word Predictor for a Federated Learning Mobile Application. TechRxiv. Preprint. https://doi.org/10.36227/techrxiv.18058682.v1

$\mathrm{DOI}$

10.36227/techrxiv.18058682.v1 


\title{
Exploring a Bilingual Next Word Predictor for a Federated Learning Mobile Application
}

\author{
N. Alfonso Burgos ${ }^{\mathrm{a}}$, K. Kišs ${ }^{\mathrm{b}}$, P. Bakarac ${ }^{\mathrm{b}}$, M. Kvasnica ${ }^{\mathrm{b}}$, G. Licitra $^{\mathrm{a}, *}$ \\ ${ }^{a}$ Neurocast B.V., Amsterdam, the Netherlands \\ ${ }^{b}$ Slovak University of Technology in Bratislava, Bratislava, Slovakia
}

\begin{abstract}
We explore a bilingual next-word predictor (NWP) under federated optimization for a mobile application. A characterbased LSTM is server-trained on English and Dutch texts from a custom parallel corpora. This is used as the target performance. We simulate a federated learning environment to assess the feasibility of distributed training for the same model. The popular Federated Averaging (FedAvg) algorithm is used as the aggregation method. We show that the federated LSTM achieves decent performance, yet it is still sub-optimal. We suggest possible next steps to bridge this performance gap. Furthermore, we explore the effects of language imbalance varying the ratio of English and Dutch training texts (or clients). We show the model upholds performance (of the balanced case) up and until a 80/20 imbalance before decaying rapidly. Lastly, we describe the implementation of local client training, word prediction and client-server communication in a custom virtual keyboard for Android platforms. Additionally, homomorphic encryption is applied to provide with secure aggregation guarding the user from malicious servers.
\end{abstract}

Keywords: Federated Learning, Bilingual Word Prediction, Character-based LSTM, Homomorphic Encryption

\section{INTRODUCTION}

In 2019, the Global Mobile Consumer Trends survey reported smartphones to be the most ubiquitous electornic device in developed markets: about $90 \%$ of consumers 5 own one and use them daily [1]. What's more, text and instant messaging apps are consumers' favorites, stirring ${ }_{30}$ a fair share of efforts into creating smoother and faster user texting experiences, i.e. auto-complete and autocorrection features, word and emoji suggestions, in-app speech-to-text, etc. For the first time, access to continuous, uninterrupted and vast amounts of data is attainable; allowing AI-driven, data-hungry algorithms to power these applications that, in turn, promote more usage and more data to be created.

Yet, most of this data is private in nature. Data privacy protection laws in the European Union - General Data Protection Regulation (GDPR) - restrict the application of AI algorithms and dictate that personal data "[..] shall be adequate, relevant and limited to what is necessary in relation to the purposes for which they are processed" (data minimisation principle) [2]. Federated Learning (FL) eliminates the need of data collection alltogether and helps develop GDPR-compliant AI systems. More specifically, FL

\footnotetext{
* Corresponding author

Email addresses: natali@neurocast.nl (N. Alfonso Burgos), karol.kis@stuba.sk (K. Kiš), peter.bakarac@stuba.sk (P. Bakarac), michal.kvasnica@stuba.sk (M. Kvasnica), giovanni@neurocast.nl (G. Licitra)
}

refers to the approach of learning a task by a federation of 25 user devices (clients) orquestrated by a central server 3 . This technique allows users to benefit from a shared global model, without the need to share data or to centrally store it. Often times, data privacy methods like differential privacy [4, 5] or homomorphic encryption [6] are embedded in the system to further improve security. FL provides with a promising alternative to server-based data collection and model training in commercial settings.

However, privacy doesn't come without costs and limitations. In commercial mobile keyboards, on-device training of language models is greatly limited. CPU usage, memory footprint, battery consumption and network bandwidth must be carefully considered when locally training and updating models in FL systems. Models are usually constraint to tens of megabytes in size to be able to run, even on high-end devices, while delivering predictions at a reasonable latency (within 20 millisenconds of an input event) [7. More often than not, these limitations come at the cost of prediciton accuracy, the size of vocabularies and multilingual capabilities.

In this paper, we investigate the possibility of a oneshot bilingual Next-Word Predictor (NWP) for a mobile application in a federated learning fashion. We simulate a FL environment where we train an RNN-based model from scratch on two languages (English and Dutch). We invesso tigate the effects of language-specific sample imbalance in learning more than a single language.

Additionally, we provide with the implementational details of the custom mobile application of our federated 
NWP. Here, model updates are performed on encrypted 55

\subsection{Related Work}

Besides data privacy benefits derived from FL by design, others also emerge in terms of resource consumption. Offloading computationally-intensive operations like 60 the training of Deep Learning (DL) models to edge devices is an incredible perk. However, due to hardware and data transfer limitations, models must be kept small and concise. State-of-the-art Language Models (LMs) are mostly self-attention based, so-called transformers, namely of tens 65 of millions of parameters, and many count with multilingual capabilibities (e.g., BERT is trained on 104 languages) [8]. Even after optimizing for size and inference latency with methods like network prunning, weight sharing, Knowledge Destillation, quantization, etc., models still re70 main on the heavy side [9]. They are suitable for on-device, offline inference but not for FL settings.

Applications of FL on Natural Language Processing (NLP) tasks have gained considerable attention in recent year [10]. Access to real-world text data, and the decen-

75 tralization of computation and storage, all while preserving privacy, acted as great incentives to this motive. Emoji prediction [11, query suggestions [12] and next-word prediction [13, 14, 7, 15] are instances of NLP tasks solved on FL environments (on-device and simulated), to name a 80 few.

Here, we focus on next-word prediction on an FL setting. Like in [13], we train a character-level RNN-based language model, except with bilingual capabilities.

\subsection{Federated Learning}

FL is an approach to distributed machine learning that builds privacy into infrastructure by design. It consist of a series of training rounds, where a central server coordinates the number of clients that participate in for a round. Each client starts off with the same global model, and computes 90 a number of local updates to it, e.g. an epoch of mini-batch stochastic gradient descent. Model updates are performed using local, client-generated training data, and are never sent to or collected centrally in the server. After training is terminated, updates are communicated to the server. The 95 server aggregates the contributions and incorporates it to the global model. Note that updates are ephemeral, and only live until immediately after aggregation. The new global model is sent back to the clients, along with the minimal information necessary for model training [3, 10, 12. A graphical representation of FL is shown in Figure 1.

Aggregation methods have been actively researched in recent years (an exhaustive overview of existing methods can be found in Tables 1 and 2 in [10). FedAvg algorithm is currently the most widespread method in FL settings, reported in over 58 publications by the end of 2020 [10]. ${ }^{125}$ FedAvg updates the server-side model using the parameters (or weights) of the model through a weighted average

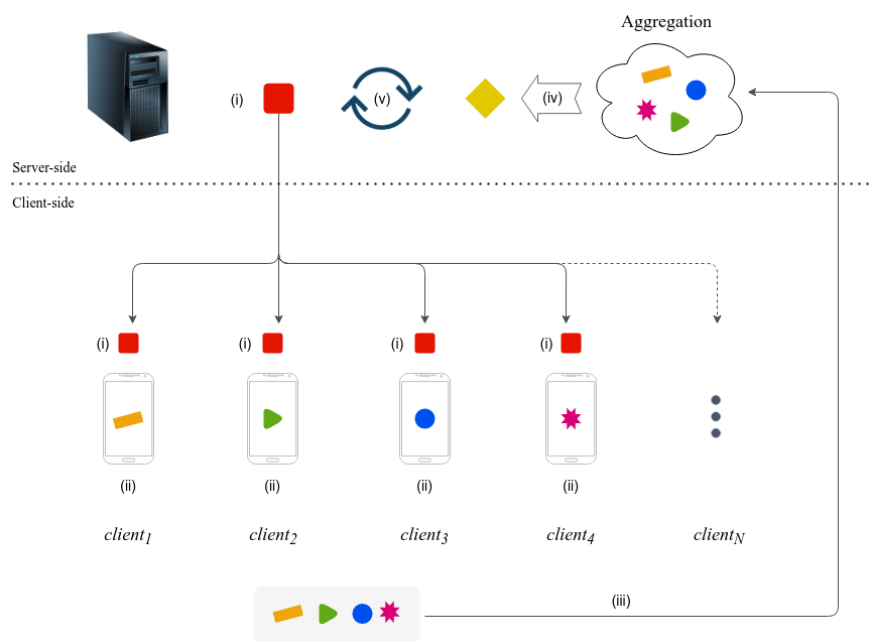

Figure 1: Federated Learning Diagram (i) Initialize global model and send them to client devices (ii) train model on client device with client data (iii) each client sends the trained model back to the server once training is complete (iv) once the number of client models for a training round are met, the server aggregates them $(\mathrm{v})$ the global model is updated and a new round of training begins.

of participating client models defined as follows

$$
w_{t+1} \leftarrow \sum_{k=1}^{K} \frac{n_{k}}{n} w_{t+1}^{k}
$$

where $w_{t+1}^{k}$ are the model parameters of the $k^{t h}$ client.

In comparison to its predecesor, FedAvg has shown to converge faster, reducing the number of training rounds by $90 \%$ and to work surprinsigly well when client models share the same initialization [3]. Because of its effectiveness and simplicity, we consider FedAvg as the FL algorithm.

Lastly, and more generally, FL is a distributed approach to an optimization problem. The implicit problem to solve in an FL setting was coined by [3] as federated optimization. Alongside communication constraints and many other practical issues, they made emphasis on the unbalanced and non Independent and Identically Distributed (IID) nature of data distributions in federated optimization. FedAvg applications showed to be robust under these conditions. In the present work, we are specially interested in non-IID distributions, not of target symbols, but across input languages. Statistical distributions of characters from English and Dutch texts are comparable, yet the effect on performance accuracy and speed of convergence of unequally distributed, language-specific texts across clients is yet to be disclosed.

\subsection{Homomorphic Encryption}

An alternative is to provide with a secure aggregation to the central averaging server that is capable of computing, at each iteration $t$, the average model $w_{t}$ in Equation (1) without explicit knowledge (hence, of interpretation) of individual client model parameters $w_{t}^{k}$. This can be 
achieved by employing the concept of homomorphic encryption [16, 17. In short, homomorphic encryption allows to perform mathematical operations over ciphers of plain-185 text data. Consider an encryption mechanism $\mathcal{E}: \mathbb{N}_{0} \rightarrow \mathbb{N}_{0}$ and a decryption function $\mathcal{D}: \mathbb{N}_{0} \rightarrow \mathbb{N}_{0}$, whereby $c=\mathcal{E}(x)$ is the cipher of a non-negative integer $x$, and $\mathcal{D}(c)=x$ is the decryption operation. Naturally, $\mathcal{D}(\mathcal{E}(x))=x$. It

135 is important to note that functions $\mathcal{E}$ and $\mathcal{D}$ are asym-190 metric; in the sense that encryption is performed using a public key that allows any interested party to generate cipher $\mathcal{E}(x)$, yet the decryption key is kept private granting permission only to its legitimate owner to apply the decryption operation $\mathcal{D}(\mathcal{E}(x))$.

Remark 1. Although the encryption and the decryption functions are defined for non-negative integers, they can easily be extended to support floating point numbers by first shifting them to the range $[0, \infty)$ and subsequently converting them to integers, e.g., by applying quantization. In what follows we assume that such a conversion, denoted by $i(x)$, was performed whenever $x$ is a floating-point number.

Unlike conventional encryption methods, homomorphic encryption allows to perform operations over ciphers. Take two non-negative integers as an example, say $a$ and $b^{205}$ Then the encryption/decryption functions $\mathcal{E}$ and $\mathcal{D}$ yield a homomorphic property if for some operation $\otimes \in\{+,-, *, /\}$ (where ' + ' stands for addition, '-' is subtraction, '*' denotes multiplication, and '/' represents division) we have that $\mathcal{D}(\mathcal{E}(a) \otimes \mathcal{E}(b))=a \oplus b$ for some $\oplus \in\{+,-, *, /\}$. If, for instance, we have $\otimes=*$ and $\oplus=+$, the product of two ciphers yields the encrypted sum of the plaintexts, i.e., $\mathcal{E}(a) * \mathcal{E}(b)=\mathcal{E}(a+b)$, which, after decryption $\mathcal{D}(\mathcal{E}(a+b))$ gives $a+b$. The obvious advantage is that one can cal160 culate the sum $a+b$ based on the ciphers $\mathcal{E}(a)$ and $\mathcal{E}(b)$ without having to decrypt them in the first place.

Various homomorphic encryption algorithms (i.e., functions $\mathcal{E}$ and $\mathcal{D}$ ) exist differing in the subset of mathe-210 matical operations in $\oplus$ and $\otimes$ they support. The socalled fully homomorphic schemes, such as SEAL [18], allow to perform any of operations $\{+,-, *, /\}$ on ciphers. On the other hand, partially homomorphic algorithms are only capable of performing a subset of operations. For ${ }^{215}$ instance, the popular RSA assymetric encryption algorithm [19] only provides the multiplicative homomorphic property, i.e., $\mathcal{E}(a) * \mathcal{E}(b)=\mathcal{E}(a * b)$. For the purpose of applying homomorphic encryption to achieving a secure central averaging, the Paillier [20] and Benaloh [21] cryp- $^{220}$ tosystems are beneficial, as both allow to calculate the encrypted sum, i.e., $\mathcal{E}(a+b)$ by multiplying the individual ciphers $\mathcal{E}(a)$ and $\mathcal{E}(b)$. In addition, the multiplication of a cipher $\mathcal{E}(x)$ by a public (i.e., not encrypted) constant $m$ can be achieved by $\mathcal{E}(x)^{m}$. Then, calculating $\frac{1}{2}(a+b)$ (i.e., the average of $a$ and $b$ ) can be achieved by considering their encrypted counterparts $\mathcal{E}(a)$ and $\mathcal{E}(b)$ and performing $(\mathcal{E}(a) * \mathcal{E}(b))^{i\left(\frac{1}{2}\right)}$, where $i\left(\frac{1}{2}\right)$ is a suitable conversion of a floating point number to an integer.
The application of the Paillier or Benaloh partially homomorphic encryption as a secure way of performing the central averaging of model parameters is self-evident. Instead of computing the average model $w_{t}$ in Equation (1) using plain-text values of $w_{t}^{k}$, each participating client encrypts its model parameters, i.e., generates $\mathcal{E}\left(w_{t}^{k}\right)$, prior to sending them to the server. The server then exploits the homomorphic property that $\mathcal{E}\left(1 / n \sum_{k} w_{t}^{k}\right)=\left(\prod_{k} \mathcal{E}\left(w_{t}^{k}\right)\right)^{i(n)}$, namely, it calculates the average of encrypted model parameters while unable to decrypt them (since it does not posses the encryption keys). The encrypted value of the average is then sent back to the clients, which are able to recover the plain text value of the average using their respective private keys. In such a way, privacy of the proposed method against malicious or dishonest central averaging servers is achieved.

\subsection{Language Model}

RNN models have been extensively used for language modeling [22]. Most work, however, is done on Long-Short Term Memory (LSTM) models; a type of RNNs that overcomes the inability of vanilla RNNs to retain long range dependencies. LSTMs use purpose-built, gated memory cells that store historical information of a sequence and ensure correct propagation of information through many time steps [23, 24, 25].

Albeit deterministic, LSTMs can be used to learn probability distributions over a sequence of language symbols. The joint probability over symbols is defined using the chain rule,

$$
\operatorname{Pr}\left(S=\left\{s_{1}, \ldots, s_{N}\right\}\right)=\prod_{i=1}^{N} \operatorname{Pr}\left(s_{i} \mid s_{1} \ldots, s_{i-1}\right)
$$

where the current symbol is conditional on the previous one, and where the context of previous symbols is encoded in the hidden states of LSTMs. The conditional probability $\operatorname{Pr}\left(s_{i} \mid s_{i-1}\right)$ is, therefore, a multinomial distribution parameterized by a softmax function.

For LMs, the goal is to maximize the log-likelihood of a given sequence of symbols by optimizing the crossentropy ( $\mathrm{CE}$ ) between the prediction and the target probabilities. However, computing the joint probability over very large number of symbols becomes prohibitively slow during training [26, 27]. Character-level language modelling is an interesting alternative to word-level or subwordlevel next-word prediction. Not only are the set of symbols much smaller in size, but allows for the inclusion of OutOf-Vocabulary (OOV) words.

To this extent, we employ a character-level LSTM with multiple layers to generate the next word. 


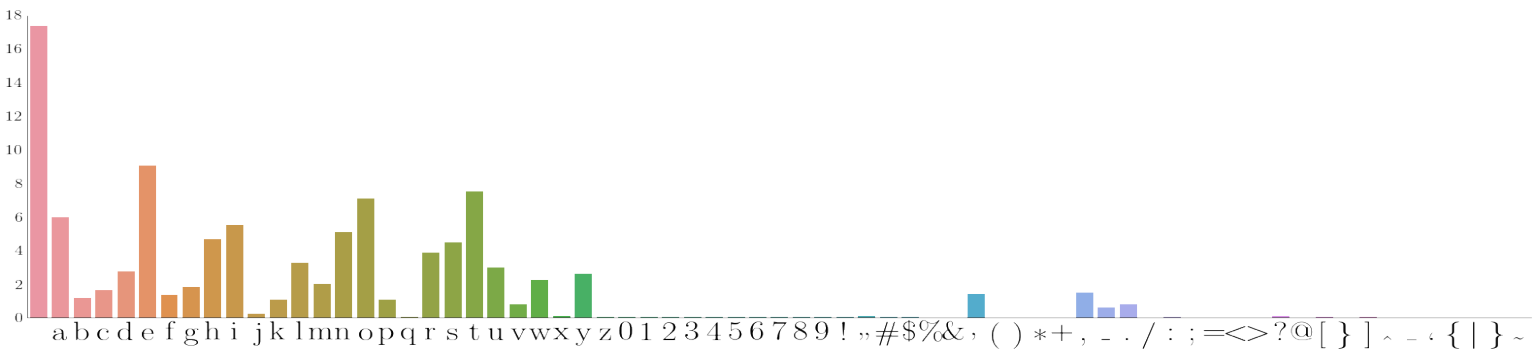

(a) English Characters

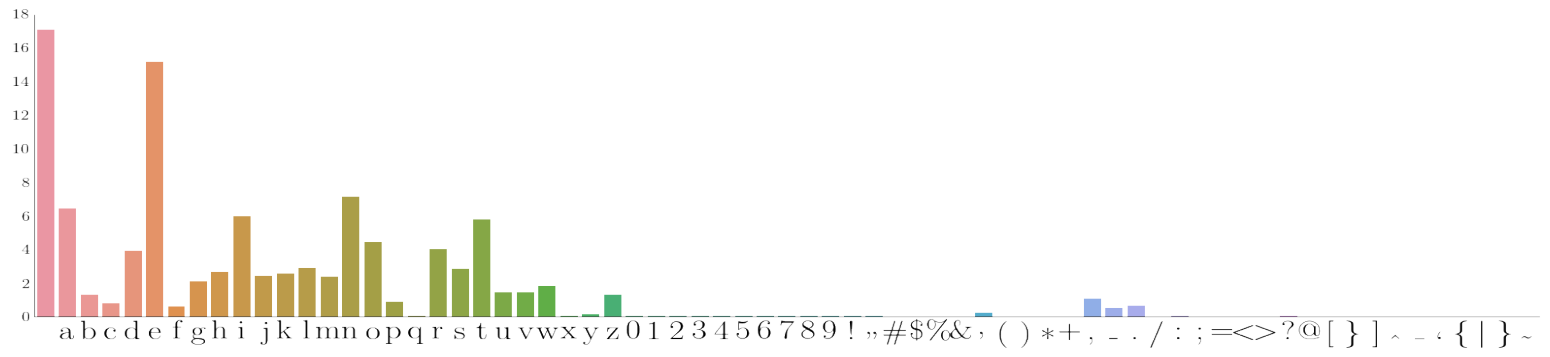

(b) Dutch Characters

Figure 2: Language Character Distributions. Character frequency distributions of (a) English and (b) Dutch texts in the training set.

\section{METHODOLOGY}

\subsection{Dataset}

The dataset is a collection of parallel corpora ${ }^{1}$ (EnglishDutch) accesible from OPUS open parallel corpus websit: $2^{2}$. Corpuses come in Moses format ${ }^{3}$ and proceed from

Corpuses are stripped of HTML tags, urls, citations, titles, subtitles, etc., and the remaining clean text is split into sentences (one per line). The aftermath is $69,7 \mathrm{M}$ lines of text data. Character frequency distributions among both 69.3 characters per sentences in English, and $92.5 \pm 72.3$

in Dutch. Sentence length variability is large and rightskewed.

\subsection{Data Partition and Distribution}

We hold out $5 \%$ of each parallel corpus as the test set. Namely, parallel sentences pertain to the same set to avoid possible lexical leakage. This results in $69,7 \mathrm{M}$ training lines and 7.7M testing lines. Note that both sets contain the same number of Dutch and English sentences.

Provided that the data is not user-tagged (at least not all of it), training samples are sharded at random and unequally into $K$ client shards to simulate an unbalanced and non-IDD client dataset. This is performed for every language in the dataset. Each client shard is further partitioned into $R$ equally-sized parts to mimic the local client cache consumed in every training round (Figure 3 .

To study the impact of language imbalance on federated optimization, the fraction $F$ of language-specific clients is experimented with. More specifically, $F$ is the fraction of English-speaking clients (EN clients) in the training data, and thus $1-F$ is the fraction of Dutch-speaking clients (NL clients). This results in $K \times F$ EN clients and $K \times(1-F)$ NL clients (Figure 8 in Appendix).

\footnotetext{
${ }^{1} \mathrm{~A}$ parallel corpus is a large and structured set of translated texts between two languages.

2 https://opus.nlpl.eu/

3 'Two aligned plain text files.

${ }^{4}$ http://www . opensubtitles.org/

${ }^{5}$ Available on http://wWW.farkastranslations.com/bilingual_ books.php
} 


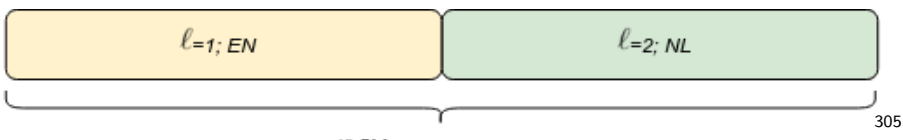

69,7M training sentences

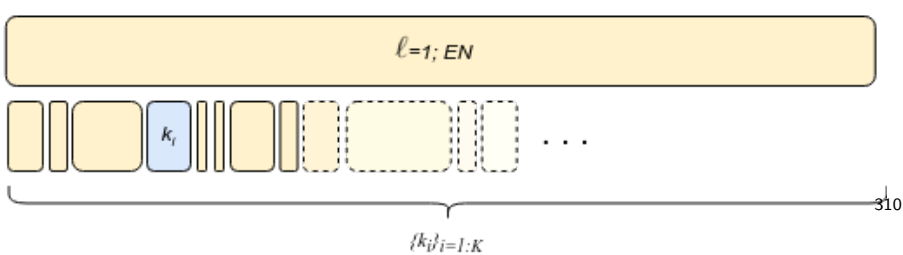

$k_{i}$

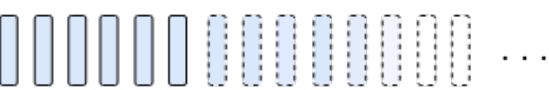

$\left\{k_{i}^{r}\right\}_{r=l: R}$

Figure 3: Client Cache Sharding. The client dataset consist of $69.7 \mathrm{M}$ English and Dutch training sentences (yellow and green), ${ }^{320}$ found at equal parts, partitioned into $K$ unequally-distributed client sets (yellow). Note that a set $\left\{k_{i}\right\}_{i=1: K}^{\ell}$ exists per language, $\ell$. In turn, local cache is simulated by sharding each client $k_{i} \in\left\{k_{i}\right\}_{i=1: K}^{\ell}$ into $R$ equally-sized parts (blue). Each client local cache $k_{i}^{r}: K \in$ $\left\{k_{i}^{r}\right\}_{r=1: R}^{\ell}$ is consumed by client model $i$ at round $r$. Glossary: number of languages $(L)$, number of clients $(K)$, number of training ${ }^{32}$ rounds $(R)$.

\subsection{Data Processing}

Given that, the Dutch and English languages employ the same (Latin) alphabet, the size of the symbol set $M=$ $|S|$ (or the vocabulary size) is reduced to $M=69$ unique characters: lowercase Latin characters (26), numbers (10), punctuation symbols (32), white-space and $\langle U N K>$ spetag. Characters in sentences are standardized to lowercased ASCII characters, and unknown characters are replaced with $\langle U N K\rangle$. Sentences are truncated at the length of 100 characters, as it has repeatedly shown the best results. Shorter sentences are pre-padded with zeros, as sug31 Lastly, characters are replaced with their index representations. Indices are obtained by enumerating the set $S$, such that $f: S \rightarrow J$, where $J=\left\{j_{1}, \ldots, j_{M}\right\}$ is the index set and $f$ is the particular enumeration of $S$.

Sentences are fed to the model as sequences of onehot character vectors. Namely, every character index, $j$, is represented by a zero-valued $M$-dimensional vector, whose element on index $j$ equals one. A language code is concatenated to the character vector. This is encoded as a binary vector of length $\left\lceil\log _{2}(\ell)\right\rceil$, where $\ell$ is the number of languages. In a bilingual setting, $\left\lceil\log _{2}(\ell)\right\rceil=1$. The resulting input sequence is of size $M+\left\lceil\log _{2}(\ell)\right\rceil=69+1=70$.

Last but not least, targets are built by forward-shifting input sequences. Essentially, any given character acts as the target to the previous character in the sequence.

\subsection{Model Architecture}

The model is a multi-layer LSTM, with six (6) stacked LSTM layers of 128 hidden neurons each. It counts with over $771 \mathrm{~K}$ parameters, and is $3 \mathrm{MB}$ in size. If weights are quantized, the model size drops as low as $0.8 \mathrm{MB}$. Very wide models (1024+ neurons per layer) with about half the depth performed better in some instances. However, with many times more the size. A deep but moderately wide model results in a more convenient trade.

\subsection{Federated Optimization}

The multi-layered LSTM is at the core of the federated next-word predictor. The Federated LSTM, or FLSTM, trains a global LSTM model distributively over $K$ clients, where only a fraction $C$ of clients are considered on each training round by the FedAvg algorithm. The global model is trained for a maximum of $R$ rounds. Clients update the global model after a training round is closed by averaging their model parameters (see Equation 1). Local training at client nodes consists of $E$ epochs of $\frac{n_{k}}{B}$ mini-batch stochastic gradient descent (SGD) optimizations with momentum, where $n_{k}$ is the number of local samples generated by the $k^{t h}$ client, and $B$ is the size of the mini-batch. Momentum is computed following Nesterov's accelerated gradient method [32]. Momentum-based optimizer have shown to work best on fully non-IID data-sets in federated learning settings [33. Additionally, to avoid exploding gradients in BPTT, gradients are clipped when their L2-norm exceeds a maximum value of 2 . This is meant to increase the robustness of model convergence.

In FedAvg, client and server learning rates are decoupled by formulating the server update as if applying SGD to the "pseudo-gradient", $-\Delta_{t}=\sum_{i}^{K}\left(w_{t}^{i}-w_{t}\right)$ [34]. This formulation is often used to increase the degree of freedom of the system for better convergence rates. However, as mentioned in 34, setting the client learning rate has a greater impact on converge. For that, and for simplicty's sake, we opt to not decouple learning rates (i.e., $\eta_{\text {server }}=1.0$ ) and tune a single (client) learning rate $\eta$. The client learning rate is decayed by

$$
\eta=\frac{\eta_{0}}{1+t_{c}}
$$

where $\eta_{0}$ is the initial value of $\eta$, and $t_{c}$ is the times $\eta$ has been decayed. Counter $t_{c}$ increases by a unit if the following condition is met

$$
\ell_{t} \leq \min _{j<t} \ell_{j}-\Delta
$$

where $\ell_{t}$ is the average training loss in round $t$, and $\min _{j<t} \ell_{j}$ is best average training loss (relative to $\Delta$ ) across an arbitrary number of previous rounds [35, 36].

\subsection{Prediction Sampling}

During inference, the next character is picked based on the joint probability over characters defined in Equation 2 Rather than selecting the character with the highest probability, one is drawn from the top- 3 most probable characters with the intention to add a higher degree of flexibility 


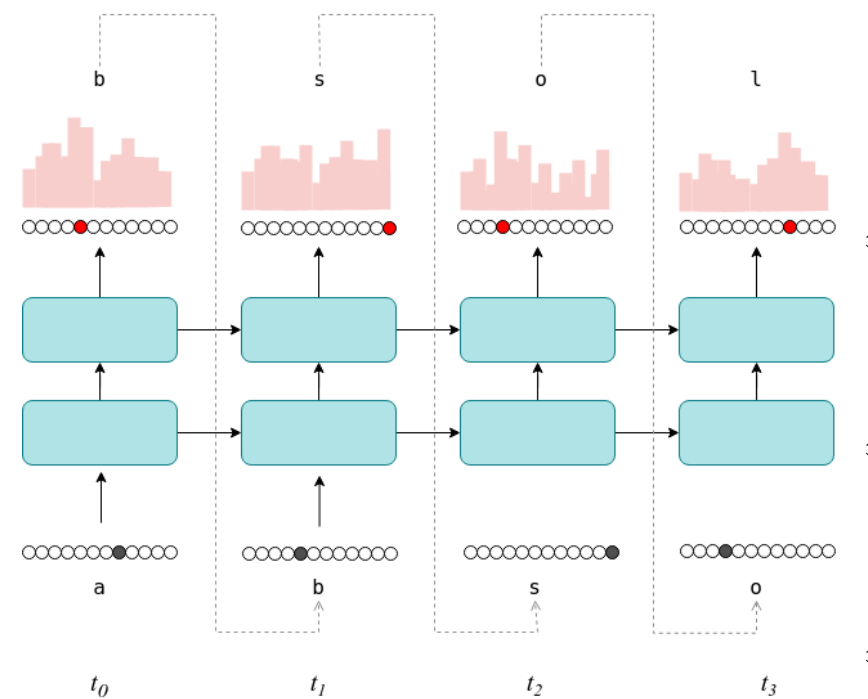

Figure 4: Top-k Prediction Sampling The model samples from the top- $k$ most probable symbols at every time step $t$.

to the language model. This is a customary strategy in language modeling (see Figure 4 as an exemplary).

The sampling process begins with a character that acts as a starts token. Start tokens can be a sequence of characters, too. At each step $t$, the model samples a charac- ${ }_{395}$ ter based on $\operatorname{Pr}\left(s_{t} \mid s_{0: t-1}\right)$. This is done iteratively until a stop symbol is encountered (e.g. white-space, $\langle U N K\rangle$, $<P A D>,<E O S>$ ) or the sequence reaches a maximum of number of characters.

During testing, this process is applied to every testing sentence independently. That is, hidden cell states are initialized at the beginning of every sentence prediction.

\subsection{Performance Evaluation}

The quality of the federated model is tested against the hold-out set described in Section 2.2. This is carried out after every training round is closed. Client models are not tested against the hold-out set: simulations are run serially on a single-GPU machine, and thus testing client models become highly time-consuming. Nonetheless, their training performance is reported.

At character-level prediction, we are interested in assessing the learning process in relation to a target performance. Central LSTM, or C-LSTM, is an LSTM trained centrally on a server, where optimization strategies like training for multiple epochs, online validation, early stopping, learning rate adaptive methods and scheduling, etc. can be applied out-of-the-box. C-LSTM yields a Cross Entropy (CE) loss of 1.37, and a overall/top-3 accuracy of $77.61 \%$ and $88.42 \%$, respectively. We use C-LSTM as the target performance for F-LSTM training. Note that C-LSTM and F-LSTM share the same model definition. Thus, we report accuracy, precision, recall and bytes-percharacter (BPC).

\section{EXPERIMENTS}

\subsection{Setup}

We simulate a F-LSTM following the methodology described above in a two-stage process: (A) the optimization of the system, which comprises the hyper-parameter tuning of the federated system to achieve the best performance and speed of convergence possible, (B) the experimentation of various ratios of EN/NL clients, or values of $F$, and their effect on performance and convergence. In this series of simulations, $F$ takes on the following ratios $F=\{0.5,0.6,0.7,0.8,0.9,1.0\}$. Note that the default language imbalance of the training data is $F=0.5$. In any case, client training and the evaluation of the global model are executed concurrently and independently.

Some parameter settings were shared across all experiments to adapt to hardware and time constraints. We simulate $K=600$ clients for a maximum of $R=1000$ rounds with a client participation ratio of $C=0.1$. Namely, $10 \%$ of clients, selected at random, are allowed to participate in every training round 6 , Learning rate $\eta$ is decayed following Equation 3 if no progress is made (relative to $\Delta=0.001$ in Equation 4 after 5 consecutive training rounds. Early stopping is implemented under the same criterion, except that training is interrupted after 15 consecutive rounds. The same seed is used for all experiments.

\subsection{Environment}

We employ an AWS EC2 instance (p2.xlarge) equipped with a single NVIDIA Tesla K80 GPU with $12 \mathrm{GiB}$ of memory, 4 vCPUs, $61 \mathrm{GiB}$ of RAM and EBS-backed storage of 100GB. Client simulations were run serially provided that we are limited to a single GPU instance.

\subsection{Results}

\begin{tabular}{ccccc}
\hline Performance Metric & \multicolumn{2}{c}{ C-LSTM } & \multicolumn{2}{c}{ F-LSTM } \\
& EN & NL & EN & NL \\
\hline CE Loss & 1.44 & 1.50 & 2.69 & 2.61 \\
Accuracy & $87.21 \%$ & $68.12 \%$ & $66.02 \%$ & $67.50 \%$ \\
Top-3 Accuracy & $93.54 \%$ & $81.75 \%$ & $70.52 \%$ & $72.82 \%$ \\
Precision & $87.21 \%$ & $75.54 \%$ & $66.02 \%$ & $67.50 \%$ \\
Top-3 Precision & $31.18 \%$ & $27.96 \%$ & $23.50 \%$ & $24.27 \%$ \\
Recall & $87.21 \%$ & $68.12 \%$ & $66.02 \%$ & $67.50 \%$ \\
Top-3 Recall & $93.54 \%$ & $81.75 \%$ & $70.52 \%$ & $72.82 \%$ \\
BPC & 2.08 & 2.16 & 17.57 & 17.05
\end{tabular}

Table 1: Table of Results. Performance metrics of best performing F-LSTM and C-LSTM with $F=0.5$. Results are reported for English (EN) and Dutch (NL), separately.

Table 1 shows various goodness-of-fit measurements of F-LSTM and C-LSTM for English and Dutch languages, separately. A performance gap stands out between centralized and federated optimization of our NWP model.

\footnotetext{
${ }^{6}$ Note that this sampling is performed keeping $F$ ratio unaltered.
} 
Train \& Test CE Loss
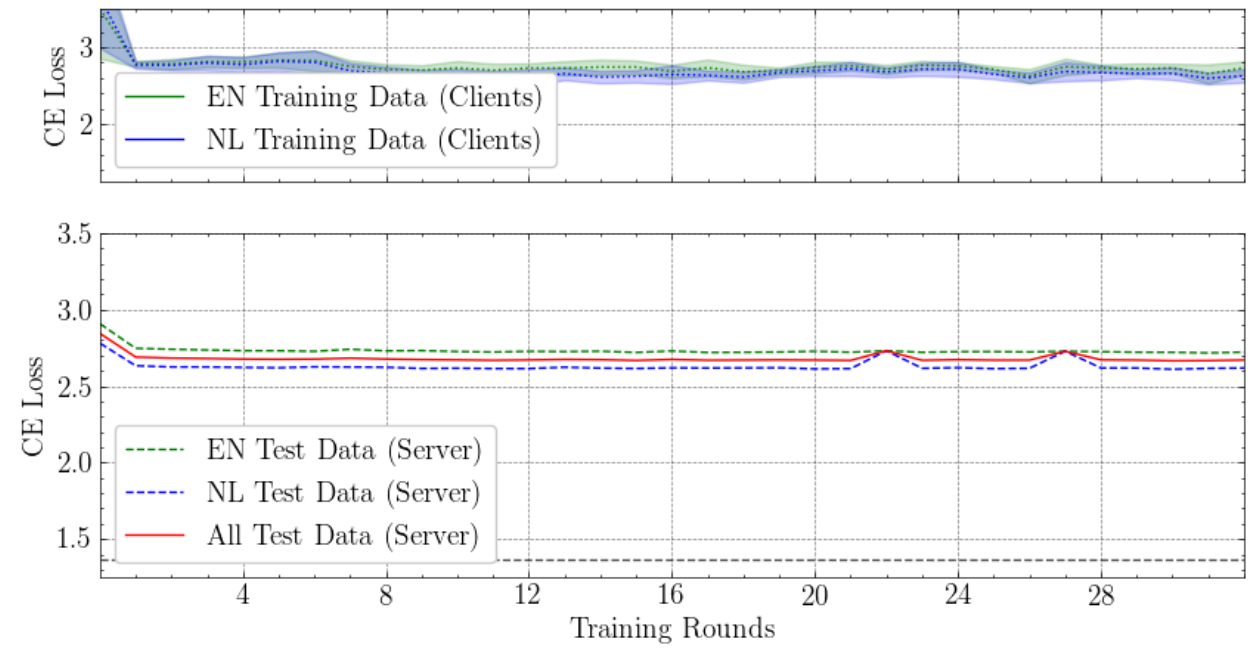

Figure 5: Train \& Test CE Loss over Training Rounds. CE loss of best performing F-LSTM for English (EN) and Dutch (NL) languages, separately. Top: Train CE loss across clients per round. Dashed lines report the average train CE loss per round. Bottom: Test CE loss per round. Overall test CE loss is outlined in solid red.

\begin{tabular}{cc}
\hline \hline Hyper-parameter & Value \\
\hline$K$ & 600 \\
$C$ & 0.1 \\
$R$ & 1000 \\
$R_{t}$ & 42 \\
$E$ & 1 \\
$B$ & 16 \\
$\eta_{0}$ & 1.5 \\
$\eta_{t}$ & 0.15 \\
$m$ & 0.9 \\
\hline
\end{tabular}

Table 2: Hyper-parameter Setting. Parameter setting of the best performing F-LSTM for $F=0.5$. Glosary: $(K)$ number of clients, $(C)$ fraction of participating clients, $(R)$ number of maximum rounds, $\left(R_{t}\right)$ convergence round, $(E)$ number of epochs, $(B)$ batch size, $\left(\eta_{0}\right)$ initial learning rate, $\left(\eta_{t}\right)$ convergence learning rate, $(m)$ momentum hyperparameter.
C-LSTM outperforms its federated counterpart by $13.98 \% 440$ in overall accuracy. Complementary to Table 1 , train and test CE loss progression over training rounds is illustrated in Figure 5 It shows that, on average, F-LSTM converges to a test CE loss of 2.82 and a top-3 accuracy of $76.2 \%$. Convergence is achieved at round $R_{t}=42$ with learning 445 rate $\eta_{t}=0.15$. Hyper-parameter settings are detailed in Table 2. These settings return the best model performance nential learning rate decay and momentum lead to a faster and more stable convergence rate, respectively. Moreover,450 Table 1 shows high rates of top-3 accuracy and recall (70\%$95 \%$ ) in both optimization cases, while precision rates recation problem, high recall and low precision suggests that there is a preference for the dominant classes (whitespace, 455 $e, a, \ldots ;$ see Figure 2, and thus the model is under-fitting the hold-out set. However, when considering the overall accuracy.

In terms of model diagnostics, Figure 5 discloses the relation between train and test CE loss. They go hand-inhand over training rounds suggesting that F-LSTM either (1) needs further training to reach the target performance,465 or (2) that it has converged to a sub-optimal local minima of the global cost function.

Figure 4 in [13] showcases the CE loss of a characterlevel NWP over 3000 training rounds during a live client evaluation of various FL settings. Readily, in the first470 rounds, it becomes evident that FedAvg plateaus showing hardly any further improvements. Yet, very high recall and precision rates were reported. It is well-known that CE loss is sensitive to label imbalance (e.g. [37]). Language character datasets for NWP are naturally and purposely imbalanced as depicted in Figure 2 2 CE loss penalizes unlikely events, even if these events are legitimately so. Hence, under a similar large CE loss many more training rounds could lead to qualitatively better models.

Alternatively, and most likely, F-LSTM has converged to an inferior solution. Theoretical understanding of the (non-)convergence of real-world applications of FedAvg is to date work in progress. Despite many efforts to tackle or minimize sub-optimality in FedAvg, [38, 35, 39, 40, 36] to name a few, there is no theoretical guarantee that it consistently improves upon mini-batch SGD local updates for non-convex objectives and heterogeneous (non-IID) settings. This is exacerbated by practicalities of FL networks, for instance, the number and selection strategy of effective devices contributing to training [36, 35].

Empirically, FedAvg has showned to be a promising estimator of the global objective [3]. Nonetheless, the performance gap between centralized and distributed training with FedAvg is particularly large for NLP tasks. This was pointed out by [38, where they compare the performance of various distributed NLP tasks with different FL algorithms and their centralized training. They report large performance gaps in accuracy across FL algorithms (Table 2 in [38]). They also noted that Fed0pt [41, where client (ClientOpt) and server (ServerOpt) optimizers are decoupled, and ClientOpt is AdamW, shows the best accuracy across the board. This is in congruence with our centralized training: adaptive optimizers worked best in comparison to vanilla and momentum SGD. Lastly, they studied the impact of different degrees of data heterogeneity in terms of label (statistical heterogeneity, non-IIDness) and quantity (sample imbalance) distribution. They showed that statistical heterogeneity has important consequences to the convergence of FedAvg. Yet, for a uniform distribu- 

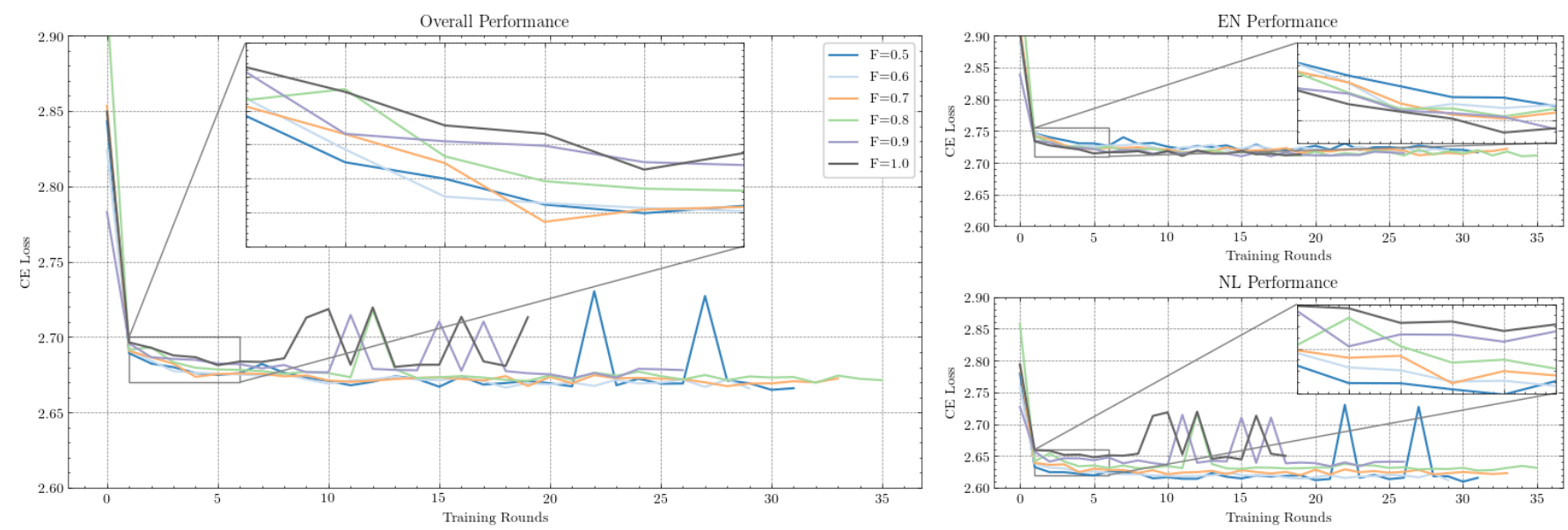

Figure 6: Test CE loss of F-LSTM with different language ratios. Test CE loss of F-LSTM for $F=\{0.5,0.6,0.7,0.8,0.9,1.0\}$. F-LSTM is tested against the overall (left), only English (upper right) and only Dutch (bottom right) hold-out sets.

tion of labels across clients, the convergence remains suboptimal for some NLP tasks (particularly, for Language Modeling tasks).

On the one hand, it is generally prefered to offload com- ${ }^{515}$ putation to client nodes, i.e. adding more local SGD updates per round, and reducing the number of communication rounds to reach a target performance. This is achieved by either decreasing $B$, increasing $E$ or $C$, or any combination of the three. Yet, allowing for more local SGD steps ${ }^{520}$ per round directs the convergence of FedAvg to an inferior optimum in non-IID settings. This is known as "client drift", and it refers to the notion that local objectives may differ drastically from the global objective. Thus, overfitting local objectives can result in biased global model ${ }^{525}$ updates. Figure 77, in the Appendix section, illustrates the degree of statistical heterogeneity (Jensen-Shanon distance) among participicating clients on the first round. Overall, clients show low statistical heterogeneity, except for a few highly heterogeneous pair of clients (in blue). We believe that these clients may be driving the drift. Mitigation strategies such as regularization [39, 40], learning rate decay schemas [36] or control variates [42, 43] can help minimize client drift, and in some cases, harnesses FedAvg to optimal convergence. All the same, in the stochastic non-convex case, convergence is not guaranteed.

We set $E=1, B=16, C=0.1$ and $R=1000$ in an attempt to find an optimal trade-off between client drift and computation efficiency, as suggested in $[3$. These settings returned $K \times C=60$ clients per round, and $3.39 \pm 2.2$ local steps in average $(61.84 \pm 35.11$ average samples per round). verge. This is in line with the intuition that smaller local step sizes have a regularizing effect while increasing gradient variance [44, 36, and in turn, mitigating client drift.530 At the risk of over-fitting, increasing $E>1$ instead accelerated convergence to the same sub-optimal point. As pointed out in [35], as long as we decay the learning rate, FedAvg can converge to the optimal even if $E>1$. The same holds for $C$. FedAvg converges to the same point535 whatever the value of $C$, which allowed for low participation and quicker training rounds. However, for large enough $\mathrm{C}$, the convergence is faster (similarly to [35]).

Differently, FedAvg is known to be robust against sample size imbalance. Recall that FedAvg computes the weighted sum of client models, where the weights are the normalized number of training samples, $\frac{n_{k}}{n}$. This allows clients to contribute to the global model proportionally to their local training data. In 38, they show that skewed sample size distribution over clients (drawn from a Dirichlet distribution for a range of $\beta$ concentration factors) do not suppose a great challenge to FedAvg. This was the case for F-LSTM, too.

\begin{tabular}{ccc}
\hline \hline Ratio of EN clients & \multicolumn{2}{c}{ Accuracy } \\
F & EN & NL \\
\hline $0.5(50 \%)$ & $66.02 \%$ & $67.05 \%$ \\
$0.6(60 \%)$ & $65.95 \%$ & $66.94 \%$ \\
$0.7(70 \%)$ & $67.77 \%$ & $66.94 \%$ \\
$0.8(80 \%)$ & $66.01 \%$ & $67.50 \%$ \\
$0.9(90 \%)$ & $5.39 \%$ & $4.90 \%$ \\
$1.0(100 \%)$ & $5.39 \%$ & $5.38 \%$ \\
\hline
\end{tabular}

Table 3: Table of Results. Performance metrics of best performing F-LSTM and C-LSTM with $F=0.5$. Results are reported for English (EN) and Dutch (NL), separately.

Last but not least, we repeatedly increase the ratio of training English clients by 10\%, and observe its effect on the convergence and performance of F-LSTM. Model accuracy for every case is collected in Table 3 and test CE losses over training rounds are depicted in Figure 6 The latter shows a decline in model performance (increase in overall CE loss) proportionally to $F$ (left). To further assess this behaviour, we tested F-LSTM against each language set, independently (bottom right: NL CE loss; top 
right: EN CE loss). As expected, NL CE loss monotonically increases with $F$, driving the overall performance decrease, while EN CE loss behaves in the opposite di- ${ }^{590}$ rection. Nonetheless, this effect is more severe in NL CE oss. This suggests that there might exist a minimum effective number of training samples or clients from where the performance stops decaying rapidly and starts improving slowly. Findings in Table 3 somewhat support this idea. ${ }^{595}$ Accuracy rates seem rather unaltered up and until a $20 \%$ ncrease in $F$, when the performance drastically plummets. Unlike CE loss comparatives in Figure 6, changes in accuracy are not gradual and complementary, but instead are abrupt and mirrored. An explanation is that the model is ${ }^{600}$ trained with the overall train CE loss, and so a decline or gain in performance in any language sub-task unavoidably affects the other. The sensitivity of performance accuracy to small changes in $\mathrm{CE}$ loss reflects the non-linear nature of their relationship for unbalanced datasets. An observa- ${ }^{605}$ tion on CE loss for performance assessment in Language Modeling tasks was mentioned earlier in this section.

\section{CLIENT-SIDE SYSTEM IMPLEMENTATION}

In this section, we provide a brief description of the proposed client-side system implementation. Inference, training and client-server communication are built within a custom soft keyboard in Android Studio.

The main feature of a soft keyboard is to test the entire framework word predictions as the user types. On-device, ${ }_{615}$ the prediction is supported by PyTorch Mobile [45] and, as the user sends or confirms the text input, the typed sequences are saved into a local cache. Predictions and text collection are disabled when the user types URIs, e-mail ${ }_{620}$ addresses, and passwords to protect the user's personal information. The reported time between the key-press and a word prediction is $11 \pm 4 \mathrm{~ms}$.

To participate in training, client devices must meet sev-625 eral requirements. The local cached data must contain a minimal number of characters. The device must be charging, idle and connected to the unmetered network. After the fullfilment of these criteria, the device is ready to train630 local model. Using Android's JobScheduler workers, we train the model and communicate with the server in the background. Training and encryption of the model are handled by Chaquopy [46]. Chaquopy is a Python635 SDK for android that enables the use of python training scripts build with PyTorch within an Android device.

A first worker, executes the local training and performs a single epoch of mini-batch stochastic gradient descent. ${ }^{640}$ Model parameters are encrypted and stored in the device. After local training is completed, a second worker sends the encrypted model parameters to the server via a POST request. Immediately after, a third worker sends a GET ${ }^{645}$ request to obtain the global model and, once received, it decrypts it.

\section{CONCLUDING REMARKS}

In this paper, we explore a bilingual next-word predictor mobile application using federated learning. First, we centrally trained a single mobile-size character-level model to predict the next word either in Dutch or in English. We show that we can successfully generate language-specific words given a language ID by continuously sampling from the model. Next, we simulate a federated learning environment to assess the feasibility of distributed training of the same model. Our results show that the federated model converges to a sub-optimal point affecting the quality of the model predictions. Longer training rounds and/or decoupling client and server optimizers and learning rates are promising options to brigde the performance gap. Furthermore, varying the ratios of training English and Dutch clients disclosed how acute language imbalance deteriorate the performance not only of one, but both sub-tasks.

Lastly, we describe the client-side implementation of the federated NWP using Pytorch Mobile, Chaquopy and Android Studio in a custom soft keyboard. We apply homomorphic encryption as a way to securely perform the central averaging of clients models against malicious or dishonest central averaging servers.

\section{References}

[1] C. Deloite, Global mobile consumer trends (2017).

[2] General data protection regulation (gdpr), https://gdpr-info. eu/ (Accessed on 18/02/2021).

[3] H. Brendan McMahan, E. Moore, D. Ramage, S. Hampson, B. Agüera y Arcas, Communication-efficient learning of deep networks from decentralized data, ArXiv e-prints (2016) arXiv1602.

[4] Y. Lu, X. Huang, Y. Dai, S. Maharjan, Y. Zhang, Differentially private asynchronous federated learning for mobile edge computing in urban informatics, IEEE Transactions on Industrial Informatics 16 (3) (2019) 2134-2143.

[5] R. C. Geyer, T. Klein, M. Nabi, Differentially private federated learning: A client level perspective, arXiv preprint arXiv:1712.07557.

[6] J. Zhang, B. Chen, S. Yu, H. Deng, Pefl: A privacy-enhanced federated learning scheme for big data analytics, in: 2019 IEEE Global Communications Conference (GLOBECOM), IEEE, 2019, pp. 1-6.

[7] A. Hard, K. Rao, R. Mathews, S. Ramaswamy, F. Beaufays, S. Augenstein, H. Eichner, C. Kiddon, D. Ramage, Federated learning for mobile keyboard prediction, arXiv preprint arXiv:1811.03604.

[8] J. Devlin, M.-W. Chang, K. Lee, K. Toutanova, Bert: Pretraining of deep bidirectional transformers for language understanding, arXiv preprint arXiv:1810.04805.

[9] Z. Sun, H. Yu, X. Song, R. Liu, Y. Yang, D. Zhou, Mobilebert: a compact task-agnostic bert for resource-limited devices (2020). arXiv:2004.02984

[10] Y. Liu, L. Zhang, N. Ge, G. Li, A systematic literature review on federated learning: From a model quality perspective, arXiv preprint arXiv:2012.01973.

[11] S. Ramaswamy, R. Mathews, K. Rao, F. Beaufays, Federated learning for emoji prediction in a mobile keyboard, arXiv preprint arXiv:1906.04329.

[12] T. Yang, G. Andrew, H. Eichner, H. Sun, W. Li, N. Kong, D. Ramage, F. Beaufays, Applied federated learning: Improving google keyboard query suggestions, arXiv preprint arXiv:1812.02903. 
[13] M. Chen, R. Mathews, T. Ouyang, F. Beaufays, Federated learning of out-of-vocabulary words, arXiv preprint arXiv:1903.10635.

[14] M. Chen, A. T. Suresh, R. Mathews, A. Wong, C. Allauzen,725 F. Beaufays, M. Riley, Federated learning of n-gram language models, arXiv preprint arXiv:1910.03432.

[15] J. Stremmel, A. Singh, Pretraining federated text models for next word prediction, arXiv e-prints (2020) arXiv-2005.

[16] C. Fontaine, F. Galand, A survey of homomorphic encryption730 for nonspecialists, EURASIP Journal on Information Security 2007 (2007) 1-10

[17] X. Yi, R. Paulet, E. Bertino, Homomorphic encryption, in: Homomorphic Encryption and Applications, Springer, 2014, pp. 27-46.

8] K. Laine, R. Player, Simple encrypted arithmetic library-sea (v2. 0), Technical report, Technical report.

[19] H. Williams, A modification of the rsa public-key encryption procedure (corresp.), IEEE Transactions on Information Theory 26 (6) (1980) 726-729.

[20] P. Paillier, Public-key cryptosystems based on composite degree residuosity classes, in: International conference on the theory and applications of cryptographic techniques, Springer, 1999 pp. $223-238$.

[21] J. D. C. Benaloh, Verifiable secret-ballot elections.

$22]$ W. De Mulder, S. Bethard, M.-F. Moens, A survey on the application of recurrent neural networks to statistical language modeling, Computer Speech \& Language 30 (1) (2015) 61-98.

[23] S. Hochreiter, J. Schmidhuber, Long short-term memory, Neural computation 9 (8) (1997) 1735-1780.

[24] F. A. Gers, J. Schmidhuber, F. Cummins, Learning to forget: Continual prediction with lstm.

[25] R. J. Williams, D. Zipser, Gradient-based learning algorithms for recurrent, Backpropagation: Theory, architectures, and applications 433 (1995) 17.

[26] R. Jozefowicz, O. Vinyals, M. Schuster, N. Shazeer, Y. Wu, Exploring the limits of language modeling, arXiv preprint arXiv:1602.02410.

[27] A. Graves, Generating sequences with recurrent neural networks, arXiv preprint arXiv:1308.0850.

[28] P. Lison, J. Tiedemann, Opensubtitles2016: Extracting large parallel corpora from movie and tv subtitles.

29] J. Tiedemann, Parallel data, tools and interfaces in opus., in Lrec, Vol. 2012, 2012, pp. 2214-2218.

[30] K. Wołk, K. Marasek, Building subject-aligned comparable corpora and mining it for truly parallel sentence pairs, Procedia Technology 18 (2014) 126-132.

[31] M. Dwarampudi, N. Reddy, Effects of padding on lstms and cnns, arXiv preprint arXiv:1903.07288.

[32] Y. E. Nesterov, A method for solving the convex programming problem with convergence rate o $\left(1 / \mathrm{k}^{\wedge} 2\right)$, in: Dokl. akad. nauk Sssr, Vol. 269, 1983, pp. 543-547.

[33] V. Felbab, P. Kiss, T. Horváth, Optimization in federated learning., in: ITAT, 2019, pp. 58-65.

[34] S. Reddi, Z. Charles, M. Zaheer, Z. Garrett, K. Rush, J. Konečný, S. Kumar, H. B. McMahan, Adaptive federated optimization (2020). arXiv:2003.00295

[35] X. Li, K. Huang, W. Yang, S. Wang, Z. Zhang, On the convergence of fedavg on non-iid data (2020). arXiv:1907.02189.

[36] Z. Charles, J. Konečný, On the outsized importance of learning rates in local update methods (2020). arXiv:2007.00878

[37] Y. Ho, S. Wookey, The real-world-weight cross-entropy loss function: Modeling the costs of mislabeling, CoRR abs/2001.00570. arXiv:2001.00570 URL http://arxiv.org/abs/2001.00570

[38] B. Y. Lin, C. He, Z. Zeng, H. Wang, Y. Huang, M. Soltanolkotabi, X. Ren, S. Avestimehr, Fednlp: A research platform for federated learning in natural language processing, arXiv preprint arXiv:2104.08815.

[39] T. Li, A. K. Sahu, M. Zaheer, M. Sanjabi, A. Talwalkar, V. Smith, Federated optimization in heterogeneous networks, arXiv preprint arXiv:1812.06127.
40] J. Wang, Q. Liu, H. Liang, G. Joshi, H. V. Poor, Tackling the objective inconsistency problem in heterogeneous federated optimization, arXiv preprint arXiv:2007.07481.

[41] S. J. Reddi, Z. Charles, M. Zaheer, Z. Garrett, K. Rush, J. Konečný, S. Kumar, H. B. McMahan, Adaptive federated optimization in: International Conference on Learning Representations, 2021.

URL https : //openreview . net/forum?id=LkFG31B13U5

[42] S. P. Karimireddy, S. Kale, M. Mohri, S. Reddi, S. Stich, A. T. Suresh, Scaffold: Stochastic controlled averaging for federated learning, in: International Conference on Machine Learning, PMLR, 2020, pp. 5132-5143.

[43] S. P. Karimireddy, M. Jaggi, S. Kale, M. Mohri, S. J. Reddi, S. U. Stich, A. T. Suresh, Mime: Mimicking centralized stochastic algorithms in federated learning, arXiv preprint arXiv:2008.03606

[44] X. Qian, D. Klabjan, The impact of the mini-batch size on the variance of gradients in stochastic gradient descent (2020). arXiv: 2004.13146

[45] A. Paszke, S. Gross, F. Massa, A. Lerer, J. Bradbury, G. Chanan, T. Killeen, Z. Lin, N. Gimelshein, L. Antiga, A. Desmaison, A. Kopf, E. Yang, Z. DeVito, M. Raison, A. Tejani, S. Chilamkurthy, B. Steiner, L. Fang, J. Bai, S. Chintala, Pytorch: An imperative style, high-performance deep learning library, in: H. Wallach, H. Larochelle, A. Beygelzimer, F. d'Alché-Buc, E. Fox, R. Garnett (Eds.), Advances in Neural Information Processing Systems 32, Curran Associates, Inc., 2019, pp. 8024-8035.

[46] Chaquopy python sdk for android, https://chaquo.com/ chaquopy/, accessed: 2021-06-10. 


\section{APPENDIX}

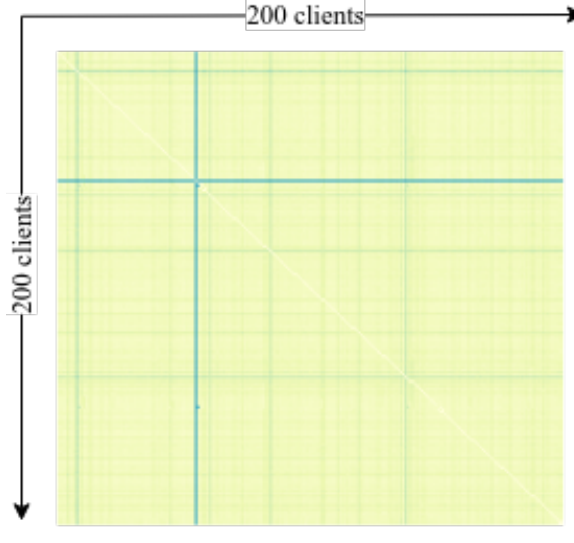

(a) English Clients

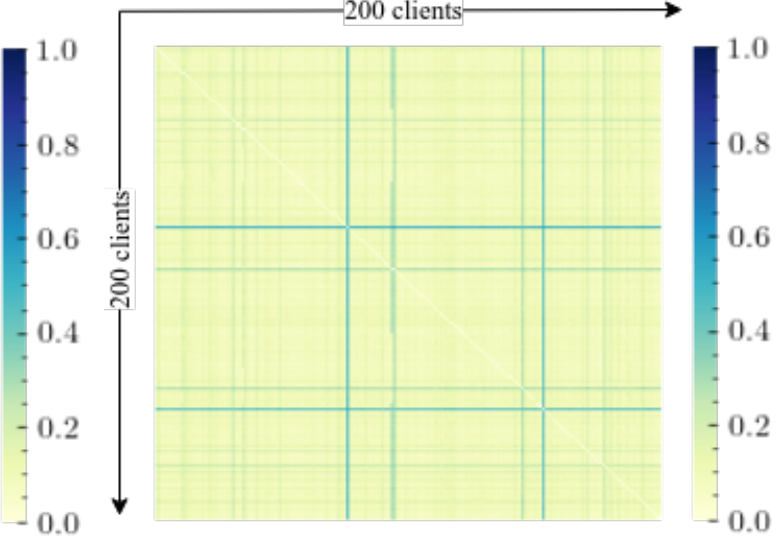

(b) Dutch Clients

Figure 7: Statistical Heterogeneity between Clients. Jensen-Shanon distance matrix between pairs of (a) 100 English clients and (b) 100 Dutch clients available in the first training round. Color intensity (more blue) represents higher level of heterogeneity
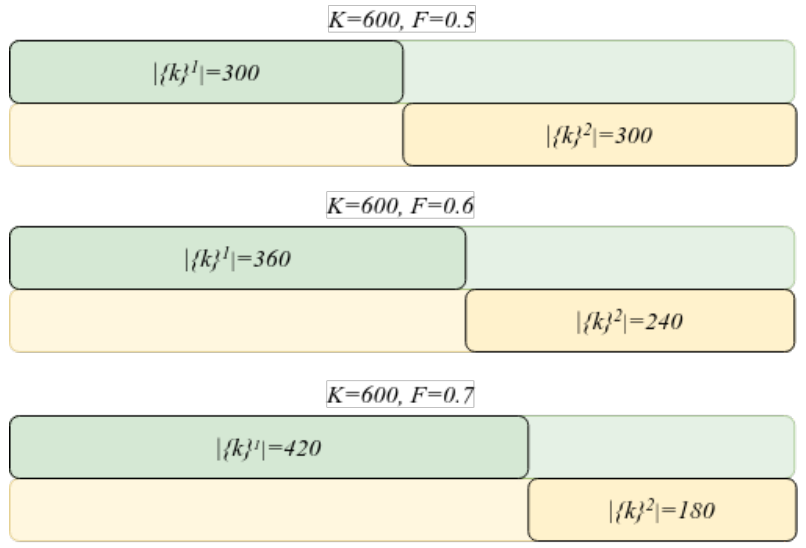

$K=600, F=0.2$

Figure 8: Language-Domain Partition. For $L=2$, where $\{k\}^{1}$ and $\{k\}^{2}$ are the set of English and Dutch clients of size $K$, respectively, and $\{k\}=\{k\}^{1} \cup\{k\}^{2}$ also of size $K$, we assign $F \times K$ English clients and $(1-F) \times K$ Dutch clients to set $\{k\}$ for any given $F$. Here, we illustrate this partition strategy for $K=600$ different values of $F$. Glossary: $(K)$ number of clients, $(F)$ fraction of English-speaking clients. 